\title{
Fibrodysplasia ossificans progressiva (FOP) presenting as a rapidly growing non-calcified neck mass
}

\author{
Ghiam Yamin $^{1,2 *}$, Shadi Daghighi ${ }^{1,3}$, Mahmood Mafee $^{1}$ \\ 1. Department of Radiology, University of California San Diego School of Medicine, San Diego, CA, USA \\ 2. Department of Neuroimaging and Neurointervention, Stanford University School of Medicine, Stanford, CA, USA \\ 3. Department of Internal Medicine, State University of New York (SUNY) Upstate Medical University, Syracuse, NY, USA
}

* Correspondence: Ghiam Yamin, MD, PhD, Department of Neuroimaging and Neurointervention, Stanford University School of Medicine, P.O. Box 19647, Stanford, CA 94309, USA

$(\triangle$ gyamin@stanford.edu)

Radiology Case. 2021 May; 15(5):10-16 :: DOI: 10.3941/jrcr.v15i5.4103

\begin{abstract}
Fibrodysplasia ossificans progressiva (FOP) is an extremely rare autosomal dominant inherited disorder leading to mature ossification within soft tissues. We report a 62-year-old female with a 3-week history of a rapidly enlarging left neck mass with no associated symptoms. A neck CT showed a $\sim 10 \mathrm{~cm}$ solid-appearing non-calcified left neck mass that markedly decreased in size on a one-month follow-up neck MRI, but with new extensive edema/intense enhancement in floor of the mouth. Prior radiographs documented hallux valgus and heterotopic ossification of the psoas/paraspinal muscles and shoulder girdle. In this case of FOP, no intervention was implemented and the symptoms improved over time and thus paralleled other such cases for flare-ups. Clinicians should be aware of this rare entity, as it is frequently misdiagnosed as cancer or other benign entities such as infection, resulting in biopsies that can often hasten disease progression.
\end{abstract}

\section{CASE REPORT}

\section{CASE REPORT}

A 62-year-old woman developed a progressive enlarging left neck mass just below the mandible that was first noticeable three weeks after discontinuation of prednisone. She was on $1 \mathrm{mg}$ of oral prednisone daily due to fibrodysplasia ossificans progressiva (FOP). She denied dysphagia, respiratory difficulties, or oral discharge. Furthermore, she did not endorse any new systemic symptoms to include recent fevers, chills, or sweats. In the interim, she was started on oral doxycycline/clindamycin and intravenous vancomycin due to possible infection. This treatment resulted in no apparent improvement. Her extensive medical history was notable for FOP, fibromyalgia, hypothyroidism (on levothyroxine), intertrigo, and muscle cramps. The patient's only notable lab value was an alkaline phosphatase level of 782 U/L (normal range: 44-147 U/L). Inflammatory/infectious markers, including white blood cell count, erthyrocyte sedimentation rate (ESR), and C-reative protein (CRP), were normal. 
Given the suspicion for a parotid mass, a neck CT with contrast was obtained, revealing a large solid-appearing noncalcified left neck mass arising between sternocleidomastoid muscle and carotid space, measuring $10 \times 3.6 \times 3.5 \mathrm{~cm}(\mathrm{CC} \times$ TV $x$ AP), compressing the internal jugular vein and displacing the common carotid and internal carotid artery medially (Figure 1). The mass was separate from the parotid gland and located posterior to the submandibular gland, extending from the angle of the mandible to the level of the clavicle without an apparent separation from the inferior aspect of the sternocleidomastoid muscle. The scout topogram from this neck CT showed no heterotopic ossification. Given these findings, differential considerations included sternocleidomastoid muscle myositis, myositis ossificans, giant angiolymphadenitis, or rhabdomyoma of the sternocleidomastoid muscle.

A one-month follow-up neck magnetic resonance imaging (MRI) revealed marked interval decreased size in the left sternocleidomastoid muscle measuring $6.7 \times 2.5 \times 2.3 \mathrm{~cm}(\mathrm{CC}$ $\mathrm{x}$ AP $\mathrm{x}$ TV), with minimal residual asymmetric prominence and enhancement (Figure 2). However, there was new extensive edema and intense enhancement involving the floor of the mouth muscles (Figure 2), including the belly of digastric muscles, myelohyoid muscles, and geniohyoid muscles. Additionally there was new submental soft tissue stranding and moderate thickening, edema, and enhancement along the lateral fibers of the lower left scalene muscle. This case is notable in regards to subsequent involvement of the floor of the mouth muscles bilaterally without involvement of the tongue muscles. Interestingly, the tongue, extra-ocular muscles, and diaphragm are spared in FOP [1].

The rheumatologist decided to monitor the patient, without restarting prednisone. Her symptoms had been stable since her neck MRI on a three-month follow-up visit, although the patient continued to endorse intermittent pain and tenderness of the neck varying by side and site. She had no difficulty swallowing but endorsed difficultly with jaw opening and chewing, similar to prior occasions, but not on the initial presentation with the neck mass.

\section{DISCUSSION}

\section{Etiology \& Demographics:}

Fibrodysplasia ossificans progressiva (FOP), previously known as myositis ossificans progressiva (MOP) and also known as Münchmeyer disease, is an extremely rare and debilitating genetic disorder of the connective tissues that is typified by skeletal malformations and progressive heterotopic ossification [1,2]. The disease is sporadic with an autosomal dominant inheritance pattern, a worldwide prevalence of $\sim 0.5$ 1 per 2 million, and a median life expectancy of $\sim 45$ years $[3,4]$.

Clinical \& Imaging Findings.

First described by John Freke in 1736 [5], FOP first manifests in early adolescence and is clinically characterized by malformation of the great toes and thumbs in addition to progressive heterotopic ossification (i.e. "secondary skeleton") of the connective tissues, including the ligaments, tendons, aponeuroses, fascia, and skeletal muscles. This ultimately results in permanent immobility and respiratory insufficiency secondary to severe scoliosis $[3,6,7]$. We report a case of a progressive enlarging left neck mass that initially presented as a clinical challenge of unknown etiology, which favored a parotid mass prior to imaging. This mass markedly improved without intervention months later, findings consistent with a FOP flare. Clinicians should be aware of this rare entity, as it is frequently misdiagnosed as cancer (e.g. sarcoma) or other benign entities, such as infection/cellulitis, resulting in biopsies that can often hasten disease progression.

FOP causes mature ossification within soft tissues, resulting in bridging between osseous structures. This typically initially affects the sternocleidomastoid muscle followed by muscles of the shoulder girdle, upper arms, spine, and pelvis [8]. The disease pattern starts with a mass, surrounding edema, and distortion of fat planes followed by heterotopic ossification in ensuing weeks and months. The ossification pattern in FOP is zonal, comprised of peripheral well-organized mature lamellar bone, intermediate osteoid bone, and a central non-ossified cellular focus (i.e. organizing granulation tissue with fibroblasts).

Radiography is typically sufficient for the evaluation of FOP $[3,6]$, which will show key characteristic disease features, including soft tissue ossification, joint malformations, malformation of the great toes (i.e. hallux valgus, monophalangic, microdactyly), malformation of the thumbs (i.e. macrodactyly), malformation of the cervical spine (i.e. facet joint fusion), malformation of the proximal femurs, and pseudoexostoses of ligamentous insertions. MRI can be useful in detecting subtle edema, characterized by $\mathrm{T} 2$ hyperintensity and enhancement on T1 post-contrast imaging, which would not be apparent on plain radiography or CT. Notably, bone scintigraphy will demonstrate activity in affected connective tissues prior to detection of heterotopic ossification on plain radiography [3]. Interestingly, a thorough retrospective chart review revealed that the patient had a hallux valgus deformity of the left great toe (Figure 3), a documented hallux deformity of the right great toe without available imaging, and a remote history of a right upper arm soft tissue mass, diagnosed as fibrosing fasciitis/myositis, that subsequently resulted in a large calcification/osseous bridge overlying the soft tissues of the right chest/shoulder (Figure 4). Pelvic radiographs from about the same time period demonstrated extensive heterotopic ossification predominantly involving the bilateral psoas muscles and right greater than left obturator muscles (Figure 5).

\section{Treatment \& Prognosis:}

No effective disease-modifying treatment exists for FOP, although corticosteroids are used for short-term treatment of flares $[1,3,9]$. The genetic underpinnings of this insidious disease have evaded investigators until recently and now upwards of 12 mutations in regions coding for the glycineserine rich and protein kinase domains of the ACVRl gene have been described [2,3,7]. ACVRI encodes activin receptor IA (ACVR1)/activin-like kinase 2 (ALK2), which is a bone 
morphogenic protein (BMP) type I receptor involved in bone formation, induction, and regeneration. Gain-of-function missense mutations of ACVRI/ALK2 ultimately result in aberrant bone formation in connective tissues and muscles [7]. A chart review revealed that genetic testing from $\sim 8$ years prior showed that the patient did not carry the c. $617 \mathrm{G}>\mathrm{A}$ germline mutation in ACVRl gene, which can be found in $\sim 90 \%$ of FOP patients. Interestingly, the patient has a sibling with FOP. As such, it is suspected that the patient is a carrier of one of the other 11 more relatively rare germline mutations that weren't tested and suggests that the patient's parents may have been carriers. As was heeded in this patient, biopsies should be avoided for suspected FOP cases as this may result in rapid bone formation in areas where tissue is removed. Furthermore, a biopsied early FOP lesion may be mistaken for several types of cancer depending on the state of lesion maturation.

\section{Differential Diagnoses:}

Due to its rarity, FOP is frequently misdiagnosed as cancer or other benign entities. A key to early diagnosis is associating a rapid waxing and waning soft tissue mass of the head, neck, shoulder, and upper back with symmetric malformed great toes [3,7]. Although biochemical testing (i.e. alkaline phosphatase or bone-specific alkaline phosphatase) may be helpful in tracking disease flares, this is not always reliable [4]. The diagnosis can only be confirmed with genetic testing of the ACVRI gene. Early diagnosis is essential to avoid unnecessary and harmful biopsies that can often hasten disease progression [10]. This is an important consideration given that patients with FOP are susceptible to ossification in regions that sustain even minor trauma. Other differential considerations include:

\section{Sarcoma:}

A reasonable differential consideration especially on initial presentation includes sarcoma (such as rhabdomyosarcoma). Sarcoma demonstrates similar edema and enhancement, marked $\left[{ }^{18} \mathrm{~F}\right]$ fluorodeoxyglucose (FDG) uptake on PET, possible associated osseous remodeling, periosteal reaction, and/or chondroid matrix. Unlike FOP, sarcoma increases in size and demonstrates persistent or increased FDG uptake on PET over time.

\section{Cellulitis:}

Cellulitis is a serious and potential common differential diagnosis to be considered in this case, especially in an older patient who is possibly more susceptible to infection. Heterogeneous enhancement, fat stranding, edema, and marked FDG uptake on PET associated with this condition will all typically return to normal after the administration of antibiotics.

\section{Dermatomyositis:}

A less common differential consideration in this case is autoimmune disease, including dermatomyositis. The edema, enhancement, and diffuse muscular FDG uptake on PET associated with this condition typically normalizes after treatment with steroids, azathioprine, and/or methotrexate. However, the soft tissue/muscular dystrophic calcifications that are typical for this condition will persist after treatment.

\section{Other:}

Alternative less likely differential considerations in this case include angiolymphadenitis/Castleman disease, aggressive fibromatosis (desmoid), malignant fibrous histiocytoma, scleroderma, and systemic sclerosis/CREST syndrome. However, none of these entities or the differential conditions listed above, is associated with symmetric skeletal malformations or waxing-waning soft tissue masses.

\section{TEACHING POINT}

Fibrodysplasia ossificans progressiva (FOP) is an infrequently encountered and thus diagnostically challenging heritable connective tissue disorder characterized by progressive heterotopic ossification that forms qualitative normal bone in muscles, tendons, and ligaments. Clinical history (i.e. rapid waxing and waning soft tissue mass of the head, neck, shoulder, and upper back) and specific quintessential radiologic signs (i.e. symmetric malformed great toes) are key to its early diagnosis and proper clinical management, including avoiding biopsies of suspected lesions that can hasten disease progression.

\section{REFERENCES}

1. Pignolo RJ, Shore EM, \& Kaplan FS. Fibrodysplasia ossificans progressiva: clinical and genetic aspects. Orphanet J Rare Dis 2011;6:80. PMID: 3253727.

2. Shore EM, Feldman GJ, Xu M, \& Kaplan FS. The genetics of fibrodysplasia ossificans progressiva. Clin Rev Bone Miner Metab 2005;3:201-204. PMID: None.

3. Kaplan FS, Le Merrer M, Glaser DL, Pignolo RJ, Goldsby RE, Kitterman JA, Groppe J, \& Shore EM. Fibrodysplasia ossificans progressiva. Best Pract Res Clin Rheumatol 2008;22:191-205. PMID: 2424023.

4. Martelli A \& Santos AR, Jr. Cellular and morphological aspects of fibrodysplasia ossificans progressiva. Lessons of formation, repair, and bone bioengineering. Organogenesis 2014;10:303-311. PMID: 4750545.

5. Peltier LF. A case of extraordinary exostoses on the back of a boy. 1740. John Freke (1688-1756). Clin Orthop Relat Res 1998; (346):5-6. PMID: 9577403.

6. Griffith G. Progressive myositis ossificans; report of a case. Arch Dis Child 1949;24:71-74. PMID: 1988209.

7. Hüning I \& Gillessen-Kaesbach G. Fibrodysplasia ossificans progressiva: clinical course, genetic mutations and genotype-phenotype correlation. Mol Syndromol 2014;5:201211. PMID: 4188166.

8. Manaster B, Petersilge C, Roberts C, Hanrahan C, \& Moore $S$ (2010) Diagnostic Imaging: Musculoskeletal: NonTraumatic Disease (Amirsys, Inc., Manitoba, Canada). ISBN: 193188479X. 
9. Glaser DL \& Kaplan FS. Treatment considerations for the management of fibrodysplasia ossificans progressiva. Clin Rev Bone Miner Metab 2005;3:243-250. PMID: None.

10. Kitterman JA, Kantanie S, Rocke DM, \& Kaplan FS. Iatrogenic harm caused by diagnostic errors in fibrodysplasia ossificans progressiva. Pediatrics 2005;116:e654-661. PMID: 16230464

\section{FIGURES}

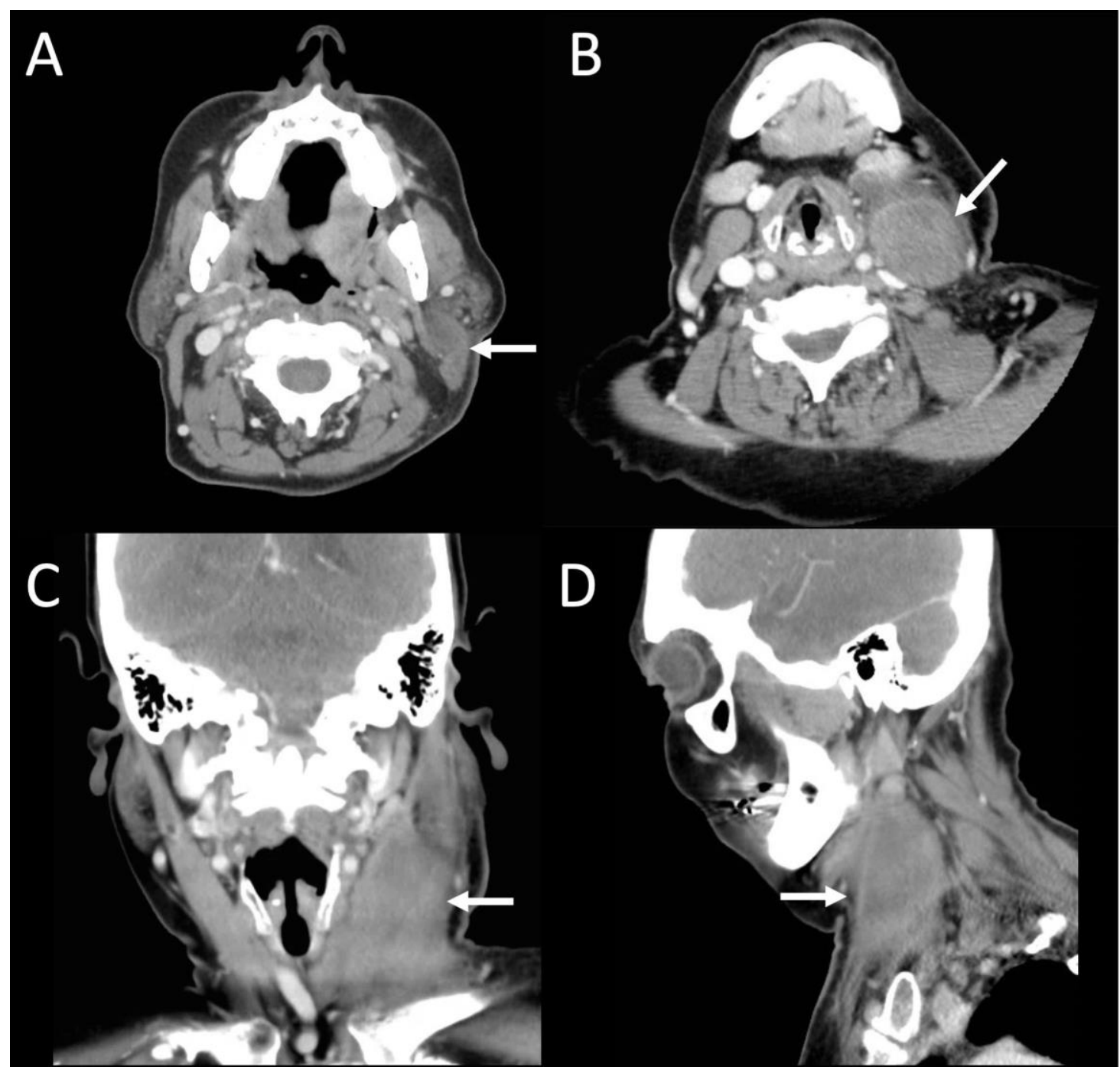

Figure 1: 62-year-old female presenting with a neck mass consistent with fibrodysplasia ossificans progressiva (FOP).

FINDINGS: Neck CT using soft tissue windowing demonstrates a large solid-appearing heterogeneously-enhancing mass arising between sternocleidomastoid muscle and carotid space measuring $10 \times 3.6 \times 3.5 \mathrm{~cm}(\mathrm{CC} \times \mathrm{TV} \times \mathrm{AP})$ as indicated by the white arrow in A. the axial plane at the level of the thyroid cartilage, B. the axial plane at the level of the parotid glands, C. the coronal plane, and D. the sagittal plane. The mass compresses and posteromedially displaces the internal jugular vein and medially displaces the common carotid and internal carotid arteries. The mass is separate from the parotid gland and located posterior to the submandibular gland, extending from the angle of the mandible to the level of the clavicle without an apparent separation from the inferior aspect of the sternocleidomastoid muscle.

TECHNIQUE: Contrast-enhanced axial CT with multiplanar reformats. Images were acquired with a GE Discovery CT750 HD CT scanner at $89.5 \mathrm{mAs}, 120 \mathrm{kV}, 5 \mathrm{~mm}$ slice thickness, and FOV 249.5, after the intravenous administration of $100 \mathrm{~mL}$ of Iohexol (Omnipaque) $350 \mathrm{mg}$ iodine $/ \mathrm{mL}$. 


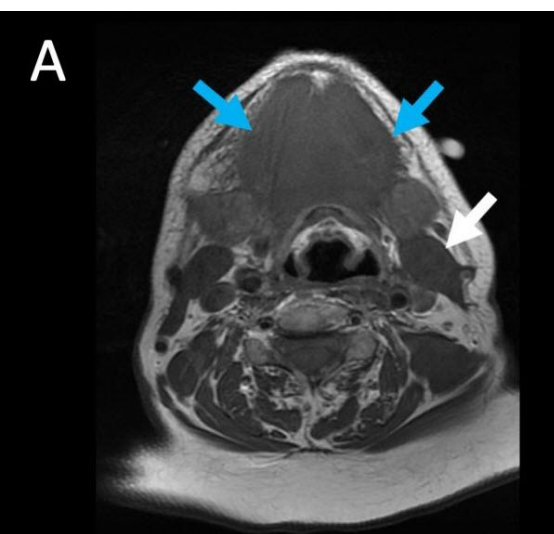

B

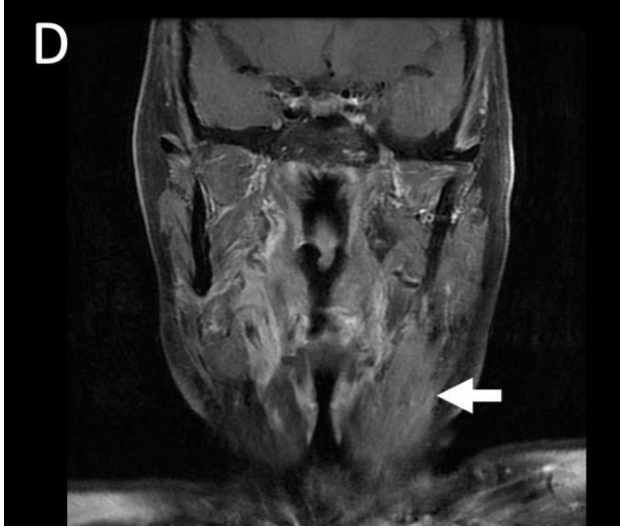

$E$
C
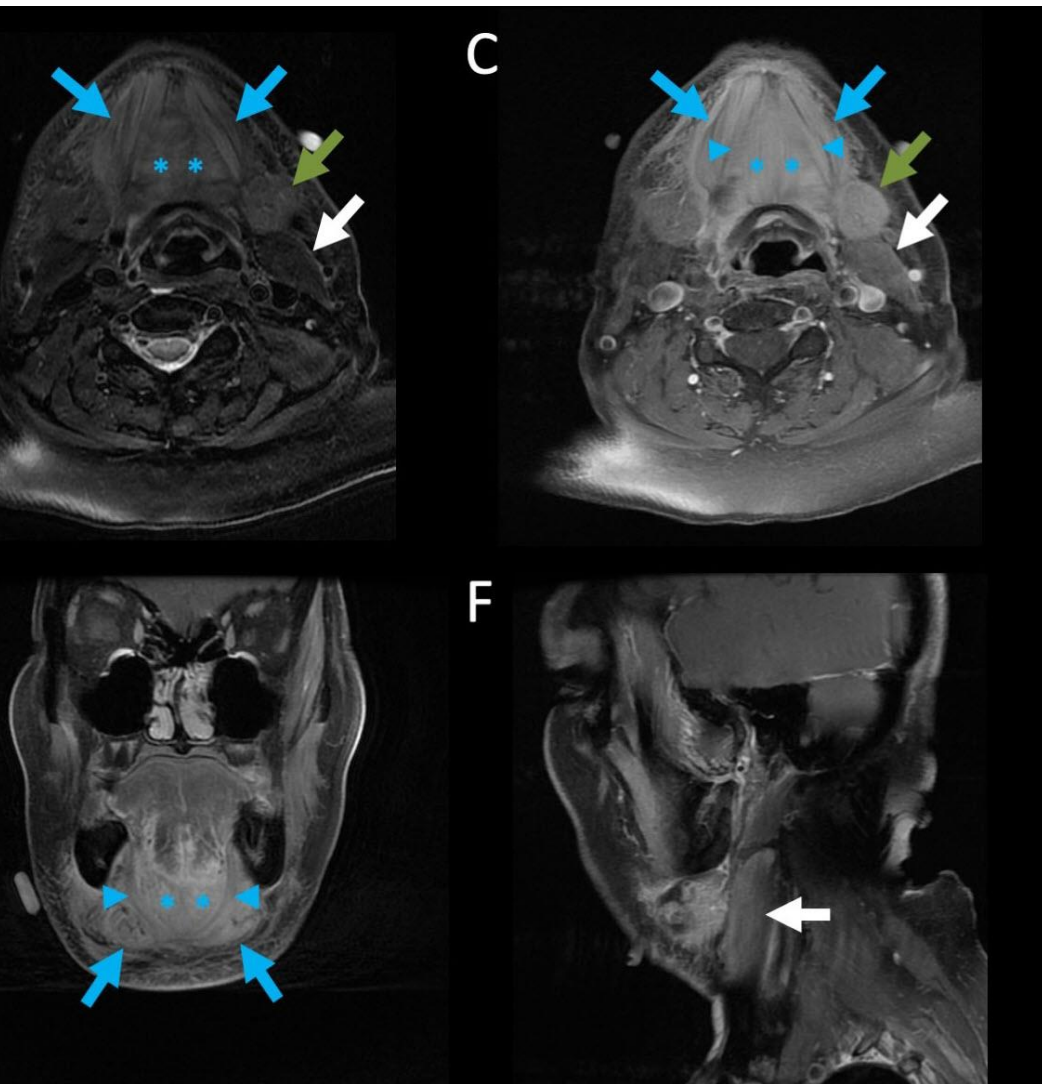

$\mathrm{F}$

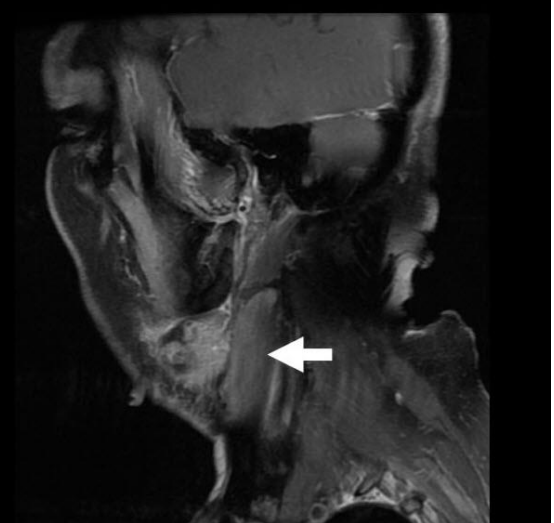

Figure 2: 62-year-old presenting with a neck mass consistent with FOP.

FINDINGS: Neck MRI highlights marked interval decreased size in the left sternocleidomastoid muscle now measuring 6.7 x 2.5 x $2.3 \mathrm{~cm}$ (CC x AP x TV), with minimal residual asymmetric prominence and enhancement (white arrows). There is new extensive edema and intense enhancement involving the floor of the mouth muscles, including the belly of digastric muscles (blue arrows), myelohyoid muscles (blue arrowheads), and geniohyoid muscles (blue asterisks), in addition to left submandibular gland with mild asymmetric edema and enhancement favored to be reactive (green arrows) on A. non-contrast axial T1-weighted MRI, B. non-contrast axial T2-weighted MRI, C. contrast-enhanced axial T1-weighted fat-saturated MRI, D-E. contrastenhanced coronal T1-weighted fat-saturated MRI, and F. contrast-enhanced sagittal T1-weighted fat-saturated MRI.

TECHNIQUE: Axial non-contrast T1-weighted neck MRI acquired on a GE Discovery MR750w 3T MRI scanner with a TE: 14.71, TR: 644, and FOV 240. Axial contrast-enhanced T1-weighted fat-saturated neck MRI acquired with same scanner at 3T, TE: 14.71, TR: 741, and FOV 240, after the intravenous administration of $6 \mathrm{~mL}$ of Gadobenate (Multihance) $529 \mathrm{mg} / \mathrm{mL}$. 


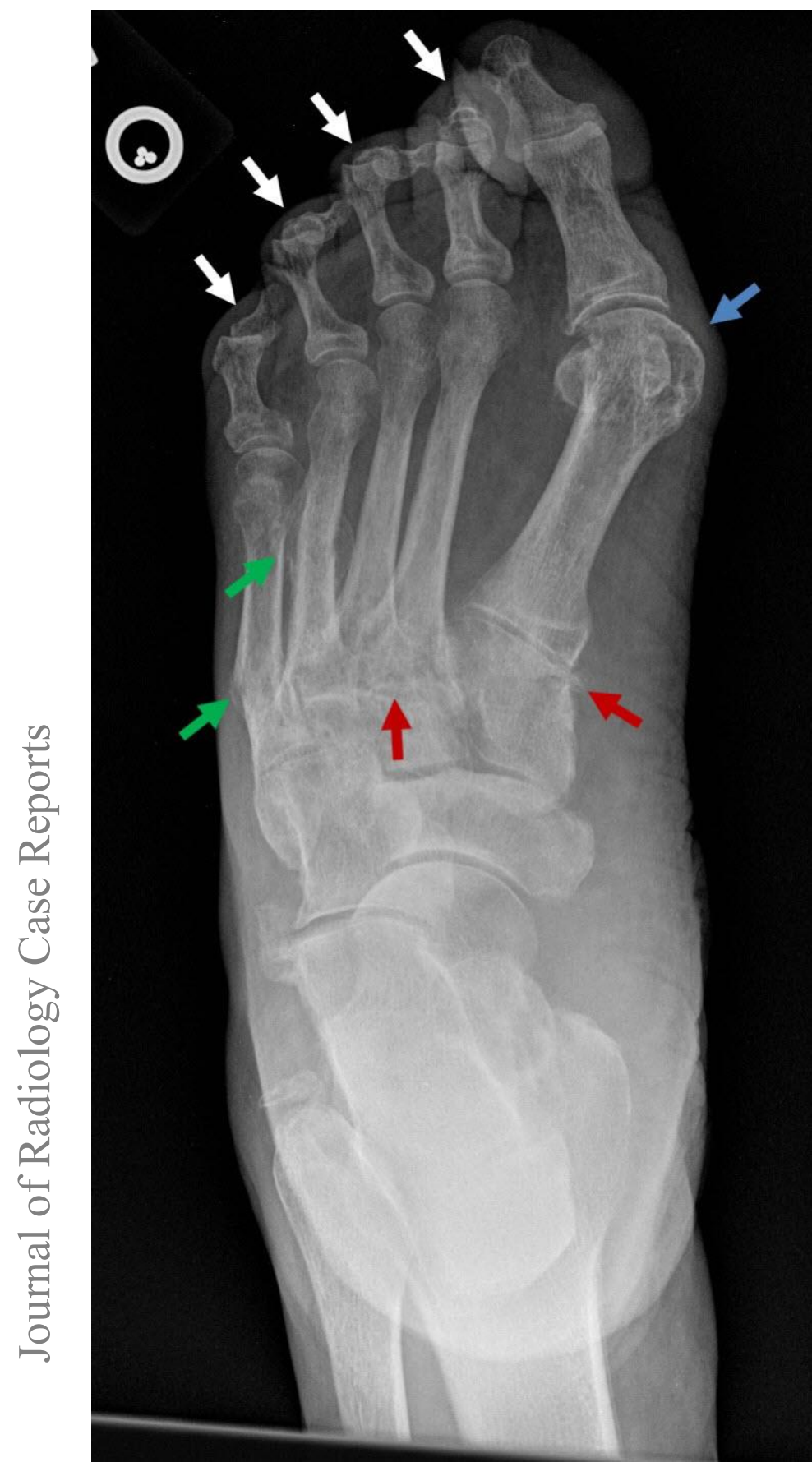

Figure 3: 62-year-old female presenting with a neck mass consistent with FOP with prior foot pain.

FINDINGS: Single AP radiograph of the left foot showing second through fifth hammertoe deformities (white arrows) and hallux valgus deformity (blue arrow). Incidentally noted chronic fracture deformities of the fourth and fifth metatarsal shafts (green arrows), in addition to polyarticular osteoarthrosis most notable at the tarsometatarsal joints (red arrows).

TECHNIQUE: Single frontal view of the left foot acquired at $55 \mathrm{kVp}$ and $4 \mathrm{mAs}$.

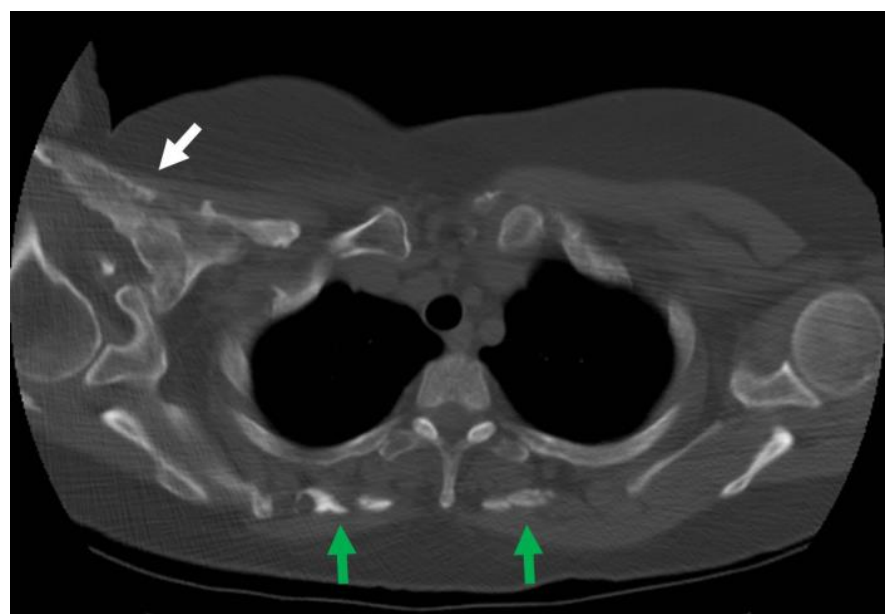

Figure 4: 62-year-old female presenting with a neck mass consistent with FOP with prior shoulder pain.

FINDINGS: Chest CT without contrast using bone windowing showing extensive calcification/osseous bridge overlying the muscles of the right chest/shoulder and bridging multiple superior ribs and the clavicle (white arrow). Heterotopic ossification noted within the soft tissues and muscles of the bilateral upper back (green arrows), including the rhomboid major.

TECHNIQUE: Non-contrast axial CT. Images were acquired with a GE LightSpeed Plus 16 Slice Helical CT scanner at $436.6 \mathrm{mAs}, 120 \mathrm{kV}, 5 \mathrm{~mm}$ slice thickness, and FOV 360.

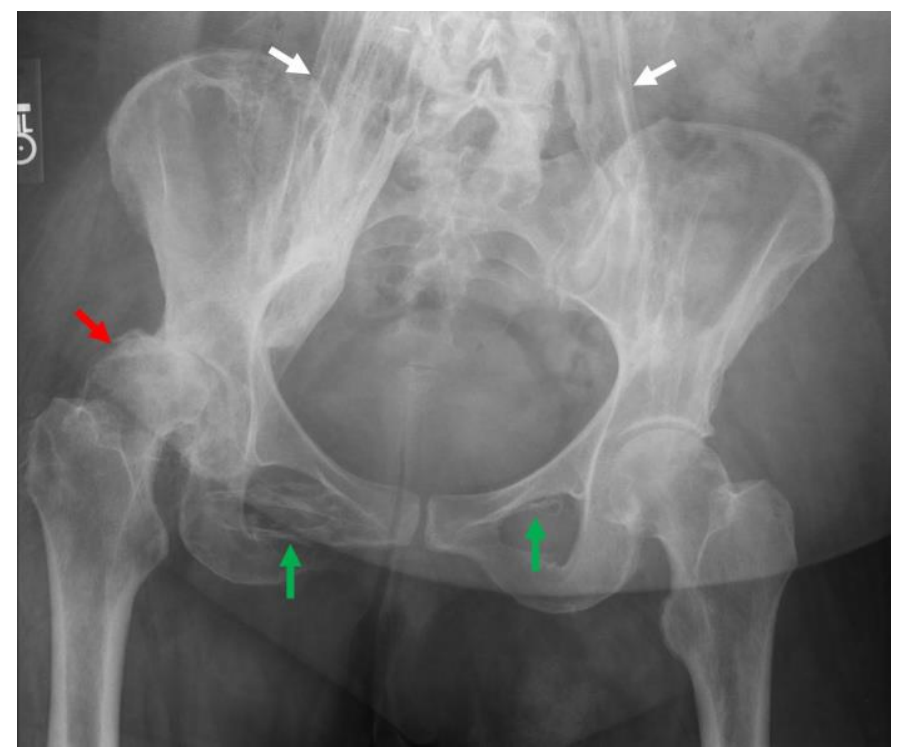

Figure 5: 62-year-old female presenting with a neck mass consistent with FOP with previous right hip pain.

FINDINGS: Single AP radiograph of the pelvis showing extensive heterotopic ossification predominantly involving the bilateral psoas muscles (white arrows) and right greater than left obturater muscles (green arrows). Severe right hip arthropathy with superolateral subluxation of the femoral head and right acetabular dysplasia (red arrow).

TECHNIQUE: Single frontal view of the pelvis acquired at 75 $\mathrm{kVp}$ and $20 \mathrm{mAs}$. 


\begin{tabular}{|l|l|}
\hline Etiology & $\begin{array}{l}\text { Gain-of-function missense mutations of } A C V R I / A L K 2 \text { resulting in aberrant bone formation in } \\
\text { connective tissues and muscles. }\end{array}$ \\
\hline Prevalence & Worldwide prevalence of $~ 0.5-1$ per 2 million. \\
\hline Gender ratio & No significant difference in gender ratio. \\
\hline Age predilection & $95 \%$ of patients manifest heterotopic ossification before age 15. \\
\hline Risk Factors & Except for family history of the disorder, no other known risk factors. \\
\hline Treatments & $\begin{array}{l}\text { No effective disease-modifying treatment exists. Corticosteroids can be used for short-term treatment of } \\
\text { flares. }\end{array}$ \\
\hline Prognosis & Median age of survival is $~ 45$ years. \\
\hline Findings on Imaging & $\begin{array}{l}\text { Radiography: Soft tissue ossification, joint malformations, malformation of the great toes/thumbs, } \\
\text { cervical spine, and proximal femurs; and pseudoexostoses of ligamentous insertions. } \\
\text { CT: Mass-like enhancement and heterotopic ossification of the soft tissues during early and late phases } \\
\text { of the disease, respectively. } \\
\text { MRI: Mass-like edema and enhancement of the soft tissues during flares. }\end{array}$ \\
\hline
\end{tabular}

Table 1: Summary table of fibrodysplasia ossificans progressiva.

\begin{tabular}{|l|l|l|l|}
\hline & CT & MRI & FDG-PET \\
\hline $\begin{array}{l}\text { Fibrodysplasia } \\
\text { progrificans }\end{array}$ & $\begin{array}{l}\text { Early stage shows mass-like } \\
\text { enhancement of soft tissues } \\
\text { whereas late stages show } \\
\text { heterotopic ossification of } \\
\text { soft tissues. }\end{array}$ & $\begin{array}{l}\text { During flare is T1 isointense to muscle, T2 } \\
\text { hyperintense, and avidly enhances. Rapid } \\
\text { waxing and waning soft tissue masses can } \\
\text { be seen depending on phase of flare. }\end{array}$ & $\begin{array}{l}\text { Marked FDG uptake during } \\
\text { flares that later normalizes } \\
\text { until the next flare. }\end{array}$ \\
\hline Sarcoma & $\begin{array}{l}\text { Heterogeneous enhancement } \\
\text { with possible associated } \\
\text { osseous remodeling, } \\
\text { periosteal reaction, and/or } \\
\text { chondroid matrix. }\end{array}$ & $\begin{array}{l}\text { T1 isointense to muscle, T2 hyperintense, } \\
\text { and avid heterogeneous enhancement. }\end{array}$ & $\begin{array}{l}\text { Marked FDG uptake that } \\
\text { persists or increases over } \\
\text { time unless treated. }\end{array}$ \\
\hline Cellulitis & $\begin{array}{l}\text { Heterogeneous enhancement } \\
\text { and fat stranding. }\end{array}$ & $\begin{array}{l}\text { T1 isointense to muscle, T2 hyperintense, } \\
\text { and heterogeneous enhancement that } \\
\text { typically returns to normal after antibiotics. }\end{array}$ & $\begin{array}{l}\text { Marked FDG uptake that } \\
\text { typically returns to normal } \\
\text { after antibiotics. }\end{array}$ \\
\hline Dermatomyositis & $\begin{array}{l}\text { Typically shows dystrophic } \\
\text { calcification in muscles and } \\
\text { soft tissues. }\end{array}$ & $\begin{array}{l}\text { Generally, T2 hyperintense and with diffuse } \\
\text { enhancement throughout affected muscles } \\
\text { that may normalize after treatment. Calcific } \\
\text { areas may be low in signal. }\end{array}$ & $\begin{array}{l}\text { Diffuse FDG uptake within } \\
\text { muscles that later } \\
\text { normalizes after treatment. }\end{array}$ \\
\hline
\end{tabular}

Table 2: Differential diagnosis table for fibrodysplasia ossificans progressiva.

\section{ABBREVIATIONS}

ACVR1 $=$ activin receptor IA

ALK2 = activin-like kinase 2

$\mathrm{BMP}=$ bone morphogenic protein

\section{Online access}

This publication is online available at: www.radiologycases.com/index.php/radiologycases/article/view/4103

\section{Peer discussion}

$\mathrm{FDG}=[18 \mathrm{~F}]$ fluorodeoxyglucose

FOP $=$ Fibrodysplasia ossificans progressiva

MRI = Magnetic Resonance Imaging

\section{KEYWORDS}

Head and neck mass; head and neck tumor; fibrodysplasia ossificans progressiva (FOP), myositis ossificans progressive (MOP); musculoskeletal syndromes; rheumatology; radiology

\section{ACKNOWLEDGMENTS}

We appreciate funding from the UCSD Clinician-Scientist Radiology Residency Program (CSRRP) (\#5T32EB00597007).
Discuss this manuscript in our protected discussion forum at: www.radiolopolis.com/forums/JRCR

\section{Interactivity}

This publication is available as an interactive article with scroll, window/level, magnify and more features.

Available online at www.RadiologyCases.com

Published by EduRad

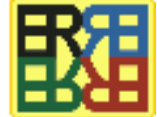

www.EduRad.org 